\title{
Comprehensive Effectiveness Evaluation of Non-lethal Sonic Weapons
}

\author{
Xianpei Jiang ${ }^{1, a^{*},} \mathrm{Li} \mathrm{Pu}^{1, \mathrm{~b}}$, Sanxue Guo ${ }^{1, \mathrm{c}}$ and Dehua Ouyang ${ }^{1, \mathrm{~d}}$ \\ ${ }^{1}$ College of Equipment Engineering, Engineering University of CAPF, Xi'an710086, China \\ a18192335913@163.com, b314576171@qq.com, c gongqingge@sina.com, d oydh2013@126.com
}

Keywords: Non-lethal; Non-lethal sonic weapons; Effectiveness evaluation; Intuitionistic fuzzy

\begin{abstract}
Concerning the uncertainty of non-lethal efficiency during using non-lethal sonic weapons, this paper introduces the mechanisms of non-lethal sonic weapons. The key factors influencing comprehensive performance of non-lethal sonic weapons are analyzed. The non-lethal performance as the core of the index system for comprehensive effectiveness evaluation is established. The intuitionistic fuzzy comprehensive evaluation has been used to improve the traditional comprehensive fuzzy evaluation, and it has been used for the comprehensive effectiveness evaluation of non-lethal sonic weapons. The evaluation theory of non-lethal weapons is feasible, and it can provide reference for the effectiveness evaluation of the other non-lethal weapons.
\end{abstract}

\section{Introduction}

Sound is a mechanical wave generated by the physical vibration, changes in the frequency and intensity cause the body to appear significant subjective feelings. Non-lethal sonic weapons utilize this kind of feature to disperse and repel offenders by directional acoustic emission [1]. According to different acoustic frequency, generally, sonic weapons are divided into Infrasound weapons, noise weapons and ultrasonic weapons. By emitting infrasonic wave below $20 \mathrm{~Hz}$, infrasound weapons make it generate resonance with human physiological systems, make the target person insane, general malaise, incapacitate his fighting capacity. Noise weapons can emit powerful sound pulses up to $150 \mathrm{~dB}$ which make target person's auditory organ appear temporary deafness, make them feel dizzy and nauseous, and lose self-control and orientation ability, thus escape from the zone of action. Ultrasonic weapons utilize high-energy ultrasonic generator to emit sonic wave with frequency above $20000 \mathrm{~Hz}$, to make strong air pressure, and cause physiological reactions such as blurred vision and nausea, resulting in fighting capacity weakened or even totally lost. Non-lethal sonic weapon can be used for the treatment of violence, riots and anti-kidnapping action; it's very effective on dispersing the crowd trouble, subduing suicide bomber and banishing terrorists hidden in shelters. Therefore, states have invested in research and development work on the sonic weapon one after another. Now the representative is the long-range directional sound dispersion device (LRAD series) which is widely used in actual combat, it is a rounded device with diameter of $80 \mathrm{~cm}$, about $20 \mathrm{~kg}$ in weight, the emitted sound intensity can be up to $150 \mathrm{~dB}$, the sound frequency ranges from 2100 to $3100 \mathrm{~Hz}$, the sonic wave can spread toward specified direction, the radius of action can reach $275 \mathrm{~m} \sim 1650 \mathrm{~m}$ [1], the weapon can be installed at the top of the armored vehicle and boat deck to disperse disorderly crowd and pirate, causing confusion among the enemy soldiers.

Non-lethal weapons have wide application prospect, but as an energy non-lethal weapon, sonic weapons are still faced with many security problems during use. First, it is relatively difficult to determine the level of non-lethal effectiveness of sonic wave, difficult to achieve the accurate control of the effective range and terminal effects of the energy, the damage to human body can not be accurately assessed, sonic energy resonating with human organs may lead to death; Secondly, the structure miniaturization is difficult. These factors have seriously restricted the development of non-lethal sonic weapons, so objective and accurate assessment of the comprehensive effectiveness of non-lethal sonic weapons is not only the needs of innovation and development of sonic weapon structure, but also the needs of operational practice and scientific application. 


\section{Establish Effectiveness Evaluation Index System}

The effectiveness evaluation of non-lethal sonic weapons is an integrated system influenced by multidimensional index with much restricted factors. To realize the purpose of dispersing and banishing the offenders, non-lethal performance is the primary index, the weapons should not cause permanent damage to living target, this is related to sound intensity, sonic wave frequency and effective range of the sonic wave; To form a strong combat effectiveness, the system should have superior technical performance, strong focusing performance, convenient energy control, and the structure should be easy to realize miniaturization; Meanwhile the weapon system should be easy to operate, reliable and cost-effective. According to these requirements, non-lethal sonic weapon effectiveness index design is shown in Fig. 1.

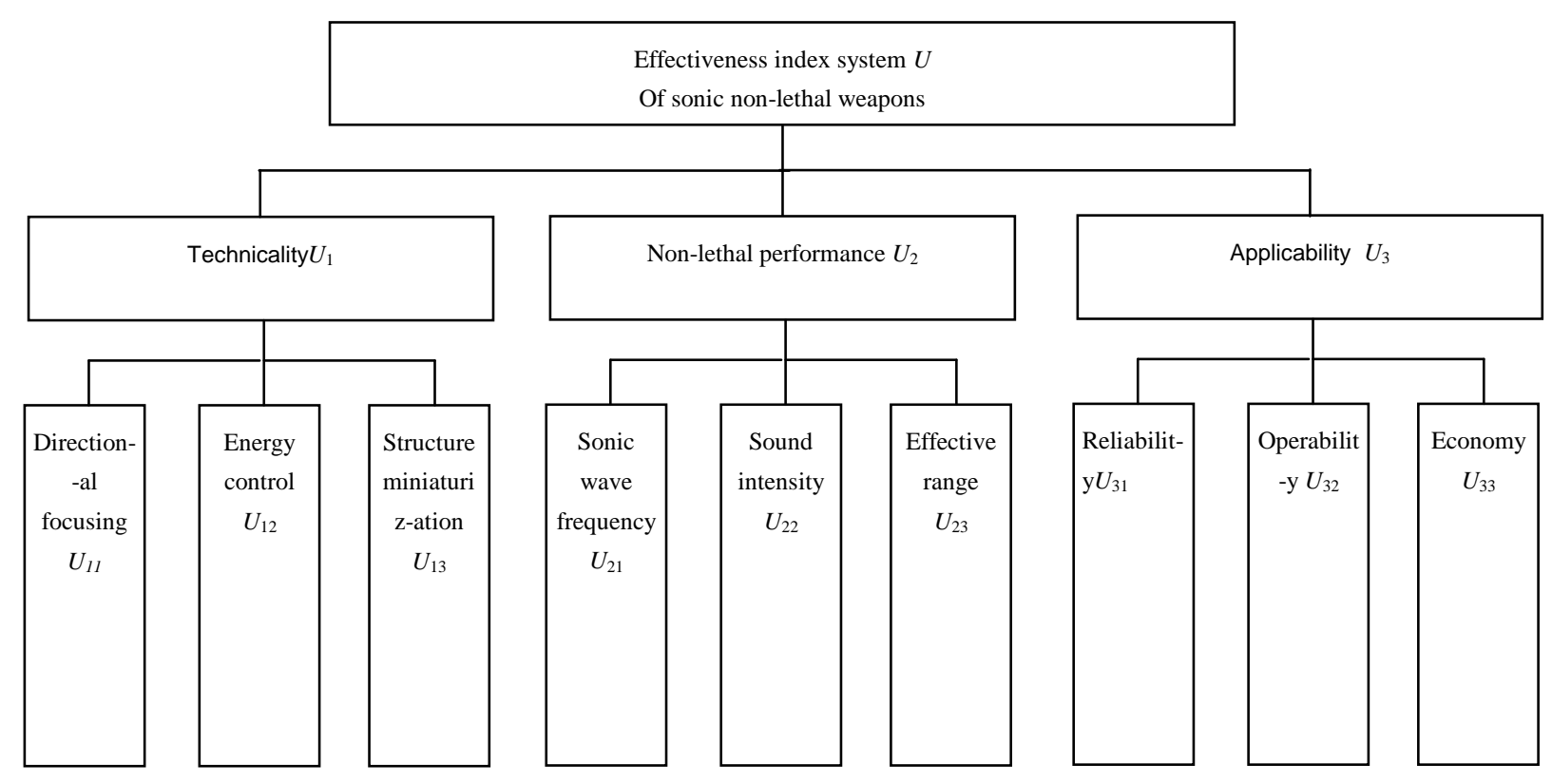

Figure 1. Index system

Technical Performance. Directional focusing. When acoustic wavelength is equivalent with the speaker's own line, acoustic radiation will be obviously directive due to sonic wave interference in space which comes from radiated from different positions of vibrating diaphragm. When infrasound sonic has longer wavelengths (e.g. When frequency is $7 \mathrm{~Hz}$, wavelength becomes 48.4 meters), it's easy to diffract and difficult to obtain high degree of directivity [2]. As a result, it'll have great impact on operator's safety; Wavelength of impulse noise is shorter than that of infrasound wave, impulse noise has better focus than infrasound wave, e.g. long-distance directional sound dissipating device can be flexibly adjusted and target rotation can be from $15^{\circ}$ to $30^{\circ}$; narrow-band ultrasonic has good direction, which is easy to control and get acoustic energy intensively.

Energy control. Regarding non-lethal sonic weapons, the key to realize directional disperse technology is the transducer, the operator control the strength of the sound element radiation sound energy by controlling acoustic frequency source output voltage volume to determine the non-lethal degree. For example, apply the planar array of Piezoelectric phased technology, the sound pressure level of a piece of PZT Piezoelectric Ceramics sound element is about 94dB [3] under 1 Pa sound pressure, the energy control of sonic weapons can be realized by controlling the sound element quantity during work.

Structure miniaturization. Generally, sonic weapons consists of three mainly parts which are sonic wave generator, power plant and control system. It's different to achieve miniaturization of sonic wave generator, for instance, the sound source diameter must reach $13 \mathrm{~m}$ [2] if generating infrasonic wave of $10 \mathrm{~Hz}$, it is essential to add parabolic reflector to make good directional spreading function, but it can only make the volume of infrasonic device bigger again; the array size of the long-distance 
sound directional disperse device named LRAD1000X is $63.5 \times 122 \times 25.4 \mathrm{~cm}$ with action distance up to $1250 \mathrm{~m}$. This shows the structure miniaturization is the development direction of sonic weapon.

Non-lethal Performance. Sonic wave frequency. Regarding the natural frequency, it is $3 \sim 17 \mathrm{~Hz}$ of human organs, $8 \sim 12 \mathrm{~Hz}$ of head, $4 \sim 6 \mathrm{~Hz}$ of abdominal viscera, the infrasonic non-lethal weapon is to choose human sensitive sonic wave frequency $4 \sim 8 \mathrm{~Hz}$ as the target [4]. Regarding impulse noise, human ear feelings on strength of different sound frequencies are different. According to equal loudness curve, $2 \mathrm{kHz} \sim 5 \mathrm{kHz}$ frequency sounds the most sensitive, so the sonic directional dispersing implement should choose the most sensitive frequency range to human ears as the attack, the emission frequency of long-distance sound directional dispersing device is $2100 \sim 3100 \mathrm{~Hz}$ which can reach a very good dispersing effect. At the same strength, the higher of the ultrasonic frequency, the greater power, and the more outstanding performance, the sonic frequency the ultrasonic gun emits can reach $30000 \mathrm{~Hz}$.

Sound intensity. The influence on body affected by sound is divided into four levels. The common sound intensity is below $120 \mathrm{~dB}$, it's influence can be restorable; for that is above $120 \mathrm{~dB}$, after a certain time of sonic impact, the inner ear are likely to suffer permanent damage; When that is high up to $150 \mathrm{~dB}$, the auditory organ will occur drum rupture bleeding, labyrinthine hemorrhage, spiral organ stripped from the basilar membrane, one stimulus is likely to make the ears completely lose the hearing [4]. The sound intensity of sonic dispersing device United States install on the armored vehicle is $145 \mathrm{~dB}$, now the sound intensity of the developed acousto-optic bomb also exceeds $140 \mathrm{~dB}$, so the sound intensity of sonic dispersing device should be controlled around 140dB.

Effective distance. The harm of sound pulse to human body depends on its energy and the distance between it with the target, a mild case is that people feel being patted or got a heavy blow; a serious case is that people will lose his breath, have a heavy headache shock and even suffocate to death [1]. For example, the long-distance directional sound dispersing device LRAD1000X can realize the voice warning within $2000 \mathrm{~m}$ range, the noise dispersing with $650 \mathrm{~m}$ range, but the maximum sound pressure level at $1 \mathrm{~m}$ reaches $165 \mathrm{~dB}$ which exceed the safe pulse SPL140dB. Therefore, the safe effective distance must be selected while using the non-lethal sonic weapons to disperse and repel offenders.

Applicability. Sonic weapons are composed of many key components, which involves many aspects such as the principle, material and production process etc. to produce high-intensive sonic wave. The sonic wave generator as the core component mainly includes information processing module and transducer module. Signal processing module adopts DSP (Digital Signal Processing) technology with high flexibility and low cost. Transducer adopts the most widely used piezoelectric transducer; the electromechanical conversion efficiency of this kind of transducer is high that usually can reach about 80\%[5], high reliability; easy to shape that can be processed into various shapes; low cost, stable performance, meanwhile, as the dispersing system application, its relatively light weight makes the maneuvering performance of equipment improved.

\section{Comprehensive Effectiveness Evaluation of Sonic Non-lethal Weapons}

According to intuitionistic fuzzy comprehensive evaluation method and evaluation index system, this paper takes the long-distance sound directional dispersing device-LRAD1000X as an example to be the evaluation object.

Intuitionistic Fuzzy Evaluation Method. Intuitionistic fuzzy set can effectively extend the traditional fuzzy comprehensive evaluation, be more flexible and effective in dealing with uncertain questions, its description of fuzzy concept is more in line with the fuzzy nature of the object, and the result is objective [6].

This paper takes five levels comment set, setting $V=\left\{v_{1}, v_{2}, \cdots, v_{5}\right\}=\{$ better, good, common, bad, worse $\}$. Adopting hundred-mark system to set $V=\{95,85,75,65,30\}$ to quantificate scores that better(90,100), good $(80,90), \operatorname{common}(70,80)$, bad(60,70), worse $(0,60)$. According to each secondary 
indexes $U_{i j}$ under index $U_{i}$, evaluation expert give intuitionistic fuzzy set of all elements in the comment set relative to sonic weapons. So the evaluation matrix is the intuitionistic fuzzy relationship between factors set and evaluation set, showed as:

$$
R=\left[\begin{array}{ccc}
r_{11} & \cdots & r_{15} \\
\vdots & r_{p q} & \vdots \\
r_{n 1} & \cdots & r_{n 5}
\end{array}\right]
$$

Among them, $n$ is the under-layer index number, $r_{p q}=\left[\mu_{p q}, \gamma_{p q}\right], \mu_{p q} 、 \gamma_{p q}$ 、 $\pi_{p q}=1-\mu_{p q}-\gamma_{p q}$ separately represent the intuitionistic fuzzy relationship, membership degree, non-membership degree and hesitation degree which are relative to the evaluation $V_{q}$ according to index $U_{p}$.

This paper uses differential correction method to make the tendentious hesitation degree value among intuitionistic fuzzy set be more in line with people's intuition, covert intuitionistic fuzzy set to the corresponding Fuzzy set [6], and compose a new evaluation matrix $R^{\prime}=\left(r_{p q}^{\prime}\right)_{n \times 5}$ composed of $r_{p q}^{\prime}$ :

$$
r_{p q}^{\prime}=\mu_{p q}+\pi_{p q} \cdot\left(0.5+\frac{\mu_{p q}+\gamma_{p q}}{2}\right)
$$

Determine the Index Weight. Based on fuzzy nine degrees scale [7], compare and quantificate each two factors in the same layer, establish fuzzy complementary judgment matrix $A=\left(a_{i j}\right)_{n \times n}, i, j=1,2, \cdots, n$. Among them, $a_{i j}$ represent the importance of index $a_{i}$ relative to $a_{j}$.

If arbitrary $i, j$ in judgment matrix $A=\left(a_{i j}\right)_{n \times n}$ satisfying condition that $0<a_{i j}<1, a_{i j}+a_{j i}=1$, then $\mathrm{A}$ is the fuzzy complementary judgment matrix. Regarding fuzzy judgment matrix $A$, $W=\left(w_{1}, w_{2}, \cdots, w_{n}\right)^{T}$ is the ordering vector of $A$ determined by minimum variance method as:

$$
\begin{aligned}
& \boldsymbol{w}_{i}=\frac{1}{n}\left(\sum_{j=1}^{n} a_{i j}+1-\frac{n}{2}\right), j=1,2, \cdots, n . \\
& B=\left(b_{1}, b_{2}, \cdots, b_{5}\right)=W \circ R^{\prime} .
\end{aligned}
$$

Referring to ten experts' experience, the fuzzy complementary judgment matrix which is relevant to the long-distance sound directional dispersing device LRAD1000X is obtained. Take the first level index as an example:

$$
A=\left[\begin{array}{ccc}
0.5 & 0.325 & 0.675 \\
0.625 & 0.5 & 0.862 \\
0.325 & 0.138 & 0.5
\end{array}\right] .
$$

It concludes the weight vector of the second level index is $W_{1}=(0.399,0.294,0.206)$, $W_{2}=(0.333,0.412,0.255), W_{3}=(0.412,0.255,0.333)$ after calculation.

Intuitionistic Fuzzy Comprehensive Evaluation to Determine Membership and Non-membership of Index. Qualitative indexes calculation. According to the evaluation index system of operational effectiveness for non-lethal sonic weapons in Fig. 1, we can find that $U_{12}, U_{13}, U_{32}, U_{33}$ are qualitative indexes. With regards to qualitative indexes, which is hard to acquire specific distribution function of intuitionistic fuzzy, so it is more suitable to use expert scoring 
method to ascertain the intuitionistic fuzzy relationship. After consulting ten experts, the intuitionistic fuzzy relationship of qualitative index relative to comment set comes to a conclusion as shown in Table 2 by the method introduced in literature [8].

Table 2 Intuitionistic fuzzy between qualitative index

\begin{tabular}{llccc}
\hline & \multicolumn{1}{c}{$U_{12}$} & $U_{13}$ & $U_{32}$ & $U_{33}$ \\
\hline$v_{1}$ & $(0.3,0.5)$ & $(0.1,0.8)$ & $(0.3,0.5)$ & $(0.1,0.8)$ \\
$v_{2}$ & $(0.4,0.4)$ & $(0.3,0.5)$ & $(0.5,0.4)$ & $(0.3,0.6)$ \\
$v_{3}$ & $(0.1,0.9)$ & $(0.3,0.5)$ & $(0.1,0.7)$ & $(0.3,0.5)$ \\
$v_{4}$ & $(0,1)$ & $(0.1,0.9)$ & $(0,1)$ & $(0.1,0.8)$ \\
$v_{5}$ & $(0,1)$ & $(0,1)$ & $(0,1)$ & $(0.1,0.9)$ \\
\hline
\end{tabular}

Quantitative Indexes Calculation. With regards to quantitative indexes, e.g. directional focusing, range of action, sound frequency, these indexes have different index dimensions, for range of action and reliability, the bigger, the better; for directional focusing, the smaller, the better; some require moderation, such as sound intensity and sonic frequency. For the need of comprehensive evaluation, it is necessary to carry out standardized treatment of indexes, to establish membership function and non-membership function of indexes relative to comment set. To calculate conveniently, we use trapezoidal intuitionistic fuzzy numbers [9] and take partial large type for illustration. Corresponding with five grades reviews, the formulas of membership function $f_{i}(x)$ and non-membership function $g_{i}(x)$ are shown from Eq. 5to Eq. 7, the graphs are shown in Fig. 2 and Fig. 3. The intuitionistic fuzzy relationship between quantitative index-relative to comment set is as shown in Table 3.

$$
\begin{aligned}
& f_{1}(x)=\left\{\begin{array}{l}
\mu_{m}, 0 \leq x \leq a_{1}, \\
\frac{b_{1}-x}{b_{1}-a_{1}}, a_{1} \leq x \leq b_{1}, \\
0, x \geq b_{1},
\end{array} g_{1}(x)=\left\{\begin{array}{l}
\gamma_{s}, 0 \leq x \leq a_{1}, \\
\frac{x-a_{1}+\gamma_{s}\left(b_{1}-x\right)}{b_{1}-a_{1}}, a_{1} \leq x \leq b_{1}, \\
1, x \geq b_{1},
\end{array}\right.\right. \\
& f_{i}(x)=\left\{\begin{array}{l}
0, x \leq a_{i-1}, x \geq b_{i-1}, \\
\frac{x-a_{i-1}}{b_{i-1}-a_{i-1}} \mu_{m}, a_{i-1}<x<b_{i-1}, \\
\mu_{m}, b_{i-1} \leq x \leq a_{i}, \\
\frac{b_{i}-x}{b_{i}-a_{i}}, a_{i}<x<b_{i},
\end{array} \quad g_{i}(x)=\left\{\begin{array}{l}
1, x \leq a_{i-1}, x \geq b_{i-1}, \\
\frac{b_{i-1}-x+\gamma_{s}\left(x-a_{i-1}\right)}{b_{i-1}-a_{i-1}}, a_{i-1}<x<b_{i-1}, \\
\gamma_{s}, b_{i-1} \leq x \leq a_{i}, \\
\frac{x-a_{i}+\gamma_{s}\left(b_{i}-x\right)}{b_{i}-a_{i}}, a_{i}<x<b_{i},
\end{array} \quad i=2,3,4,\right.\right. \\
& f_{5}(x)=\left\{\begin{array}{l}
0,0 \leq x \leq a_{4}, \\
\frac{x-a_{4}}{b_{4}-a_{4}}, a_{4}<x<b_{4}, \\
\mu_{m}, x>b_{4},
\end{array} \quad g_{5}(x)=\left\{\begin{array}{l}
1,0 \leq x \leq a_{4}, \\
\frac{b_{4}-x+\gamma_{s}\left(x-a_{4}\right)}{b_{4}-a_{4}}, a_{4}<x<b_{4}, \\
\gamma_{s}, x \geq b_{4},
\end{array}\right.\right.
\end{aligned}
$$

In these formulas, $\mu_{m}$ and $\gamma_{s}$ respectively represents the maximum membership and the minimum non-membership of intuitionistic fuzzy numbers. Considering most actual situation and intuitive experience, we value that $\mu_{m}$ is 0.8 and $\gamma_{s}$ is 0.1 [6]. 


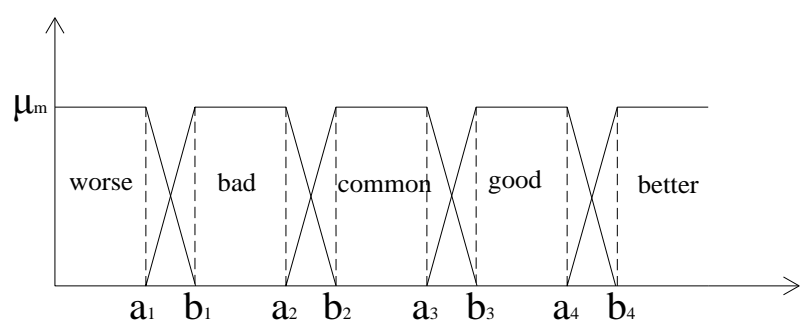

Figure 2. Membership function of the bigger the better

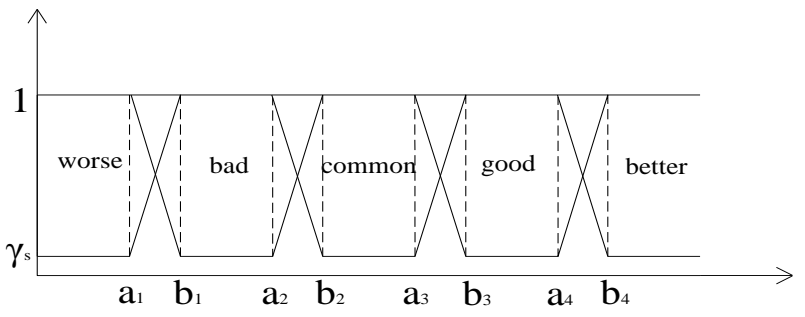

Figure 3. Non-membership function of the bigger the better

Table 3 Intuitionistic fuzzy between quantitative index

\begin{tabular}{llllll}
\hline & \multicolumn{1}{c}{$U_{11}$} & \multicolumn{1}{c}{$U_{21}$} & \multicolumn{1}{c}{$U_{22}$} & \multicolumn{1}{c}{$U_{23}$} & $U_{31}$ \\
\hline$v_{1}$ & $(0,1)$ & $(0,1)$ & $(0.26,0.72)$ & $(0,1)$ & $(0.20,0.73)$ \\
$v_{2}$ & $(0.4,0.55)$ & $(0.30,0.66)$ & $(0.64,0.28)$ & $(0.73,0.18)$ & $(0.75,0.10)$ \\
$v_{3}$ & $(0.4,0.55)$ & $(0.50,0.43)$ & $(0,1)$ & $(0.08,0.91)$ & $(0,1)$ \\
$v_{4}$ & $(0,1)$ & $(0.1,0.9)$ & $(0,1)$ & $(0,1)$ & $(0,1)$ \\
$v_{5}$ & $(0,1)$ & $(0,1)$ & $(0,1)$ & $(0,1)$ & $(0,1)$ \\
\hline
\end{tabular}

Comprehensive evaluation:

$$
B=W \circ R^{\prime}=(0.333,0.496,0.154) \circ\left[\begin{array}{ccccc}
0.175 & 0.58 & 0.19 & 0 & 0 \\
0.171 & 0.61 & 0.18 & 0 & 0 \\
0.379 & 0.422 & 0.25 & 0.05 & 0.03
\end{array}\right]=(0.202,0.56,0.19,0.008,0.005)
$$

If it is expressed in hundred-mark system, the score in comprehensive evaluation of the sonic weapons is

$E=B \cdot V^{T}=81.7100$,

Using the same procedure, the score in comprehensive evaluation of the infrasonic weapons is

$$
E_{1}=B_{1} \cdot V_{1}^{T}=75.39 \text {, }
$$

The score in comprehensive evaluation of the ultrasonic weapons is

$$
E_{2}=B_{2} \cdot V_{2}^{T}=78.85 \text {. }
$$

Based on the calculation analysis on the comprehensive effectiveness of long-distance sound dispersing device, infrasonic weapons and ultrasonic weapons, and also the comment results, it concludes that the comprehensive effectiveness evaluation of intuitionistic fuzzy comprehensive evaluation, the results show that the evaluation for comprehensive effectiveness of long-distance sound dispersing device is good, and that of infrasonic weapons and ultrasonic weapons is ordinary.

\section{Conclusion}

In this paper, the current development situation and battle practice of non-lethal sonic weapons are considered comprehensively. It establishes the effectiveness evaluation index system of non-lethal sonic weapons, which takes technicality, non-lethal performance and applicability as the first grade index, takes directional focusing and structure miniaturization as the second index. This index system can reflect the non-lethal comprehensive performance of sonic weapons objectively.

The intuitionistic fuzzy comprehensive evaluation method has been used to improve the traditional one, it provides more flexibility and practicability on dealing with fuzziness, uncertainty and more, 
the description of fuzziness concept can fit with objective's fuzziness nature better, this method of evaluation has a strong theoretical property, its process is simple and its results are credible and close to reality.

Separately evaluate the comprehensive effectiveness of long-distance sound directional dispersing device, infrasonic guns and ultrasonic guns. It shows from the results of evaluation that the common technical problem of sonic weapons is to realize miniaturization on structure, because the realization of structure miniaturization will affect the directional focusing and intensity of acoustic beam. The long-distance sound dispersing device has been widely used on actual combat as it has realized effective control on energy and good directional focusing in technology; Concerning infrasonic weapons, it's very difficult to produce infrasonic wave and also hard to control the infrasonic wave, so the technical problem of infrasonic weapons is the difficulty on acoustic beam focusing and strength of acoustic beam is low. Concerning ultrasonic weapons, as the ultrasonic wave is easy to attenuate in the air, so its effective operating distance is short.These are the main factors limiting all kinds of sonic weapons in battle practice, which are also the key points in future research.

\section{References}

[1] Bo ZHANG, Yan-feng LIANG. Research on Development of Non-fire Strike Weapons [J]. Journal of CAEIT, 2015, (1):10-11.

[2] De ke LI. Study on the theory of acoustic frequency directional transmission system based on acoustic parametric array and the application research [D]. Chengdu: University of Electronic Science and Technology, 2011:1-14.

[3] Xingjuan LIU. Study on Piezoelectric Infrasonic Phased Array Technology [D]. Taiyuan: North University of China, 2014:5-28.

[4] Yulan DING. Man-Machine Engineering [M]. Version fourth. Beijing: Beijing Institute of Technology Press, 2015:159-164.

[5] Ying jie LEI, ZHAO Jie, Zheng hong HE, et al. Intuitionistic Fuzzy Set Theory and Applications [M]. Beijing: Science Press, 2014:28-51, 191-200.

[6] Zhongmin Li, Shuchun Tang, Li Jun, Chen Jian. Study on Acquisition Risk Identification for Weapon and Equipment Based on the Full Life Cycle [J].Engineering Science, 2005, 7(12):49-53.

[7] Guiyuan YANG, Jili HUANG. Mathematical Modeling [M]. Hefei: University of Science and Technology of China Press, 2008.

[8] Haiming YANG, Yue LI, Weicheng KONG, et al. The application of the DELPHI method to intuitionistic fuzzy comprehensive evaluation [J]. Mathematics in Practice and Theory, 2012, 42(23): 169-172.

[9] Xubo WANG, Sijun BAI, Libiao BAI, et al. Attribute collaboration of aerospace project portfolio allocation based on intuitionistic trapezoidal fuzzy number [J]. Journal of Astronautics, 2014, 35(12): 1396-1404. 